\title{
Generation and excitation of point defects in silica by synchrotron radiation above the absorption edge
}

\author{
F. Messina, * L. Vaccaro, and M. Cannas \\ Dipartimento di Scienze Fisiche ed Astronomiche, Università di Palermo, Via Archirafi 36, I-90123 Palermo, Italy
}

(Received 1 July 2009; revised manuscript received 19 November 2009; published 28 January 2010)

\begin{abstract}
We report photoluminescence measurements carried out on amorphous $\mathrm{SiO}_{2}$ upon excitation by synchrotron light. Exposure of the as-grown material to above-edge light at low temperature induces the formation of nonbridging oxygen hole centers (NBOHC), localized in a thin layer below the surface limited by the penetration depth (tens of $\mathrm{nm}$ ) of impinging light. After concluding the exposure to $11 \mathrm{eV}$ light, stable defects are revealed by observing their characteristic $1.9 \mathrm{eV}$ photoemission band excited at $4.8 \mathrm{eV}$. The local concentration of induced defects, supposedly formed by nonradiative decay of excitons, is very high (close to $\sim 10^{21} \mathrm{~cm}^{-3}$ ) and independent of the previous history of the material. On the other side, we also observe the $1.9 \mathrm{eV}$ emission upon excitation between 9 and $14 \mathrm{eV}$, accompanied by the $2.5 \mathrm{eV}$ luminescence ascribed to self-trapped excitons. This $1.9 \mathrm{eV}$ band exhibits a temperature dependence different from that measured by excitation within the absorption bands at 4.8 and $6.4 \mathrm{eV}$ of $\mathrm{NBOHC}$, and is proposed to arise from the fact that $\mathrm{NBOHC}$ are generated by above-edge light in their excited electronic state.
\end{abstract}

DOI: 10.1103/PhysRevB.81.035212

PACS number(s): 61.80.Ba, 71.55.Jv, 78.55.Qr, 41.60.Ap

\section{INTRODUCTION}

Amorphous silicon dioxide $\left(\mathrm{SiO}_{2}\right)$, or silica, is an insulator playing an archetypal role in current physics of amorphous solids, as well as a material widely used in applications including optical fibers, optical components for photolithography, and metal-oxide-semiconductor transistors. The wide applicability of this solid is vastly founded on its high optical transparency extending from the infrared up to the vacuum ultraviolet (VUV) absorption edge, located at $E_{0} \sim 8.5 \mathrm{eV}^{1-3}$

The optimal features of silica are limited by the formation of point defects, usually induced by exposure to ionizing or high-power laser radiation and giving rise to wide absorption bands limiting the transparency range. ${ }^{1-4}$ The two most common point defects in silica, virtually responsible of the whole UV absorption of irradiated high purity silica specimens, are the paramagnetic dangling bonds localized on $\mathrm{Si}$ (also known as $E^{\prime}$ center) and on $\mathrm{O}$ [nonbridging oxygen hole center (NBOHC)] atoms. ${ }^{1-4}$ The microscopic structure of these two defects can be represented as $\equiv \mathrm{Si}^{\circ}\left(E^{\prime}\right.$ center $){ }^{5}$ and $\equiv \mathrm{Si}-\mathrm{O}^{\circ}(\mathrm{NBOHC}){ }^{6,7}$ where the $\equiv$ symbol stands for three back bonds with oxygen atoms, while $\cdot$ represents an unpaired electron. While the optical activity of $E^{\prime}$ center consists of an absorption band peaked at $5.8 \mathrm{eV},{ }^{1,4}$ that of $\mathrm{NBOHC}$ is more complex. Indeed, the latter gives rise to two absorption bands in the UV range, peaked at 4.8 and $6.4 \mathrm{eV}$, accompanied by a much weaker band at $2.0 \mathrm{eV}^{.8-11}$ All the three absorptions excite a long-living $(10 \mu \mathrm{s})$ emission at $1.9 \mathrm{eV}$ which is the most distinctive feature of this defect. ${ }^{7}$ NBOHC is currently studied as an important model defect having some almost unique features among defects in oxides related to its weak coupling with the vibrations of the matrix. ${ }^{12-15}$

While the detrimental influence of point defects on the performance of silica in applications is a well established fact, defects are still the subject of ongoing research aimed to clarify several poorly understood aspects of their generation processes, the factors determining their stability or instability, as well as some of their most basic spectroscopic properties. In regard to the formation mechanisms, while several works have investigated the generation of $E^{\prime}$ and NBOHC centers induced by high-power laser (up to $7.9 \mathrm{eV}$ photon energy) or ionizing radiation, ${ }^{16-26}$ little information is available on possible generation of these defects upon exposure of $\mathrm{SiO}_{2}$ to low power above-edge VUV radiation.

Although the position $E_{0} \sim 8.5 \mathrm{eV}$ of the edge can be operatively defined by VUV absorption measurements, the physical interpretation of the electronic transitions responsible for absorption above $E_{0}$ is still debated, and the same occurs for the value of the band-gap energy, reported by different authors to be either near $9 \mathrm{eV}$ or near $11 \mathrm{eV}^{1,27-29}$ While silica shows measurable photoconductivity starting from $8.9 \mathrm{eV}$, photoconductivity and photoemission signals markedly increase only above $11 \mathrm{eV}$, this being accompanied by qualitative variations of the photoelectron energy distribution curve. ${ }^{1,27}$ Hence, it has been argued that the observed photoemission below $11 \mathrm{eV}$ was due to secondary processes and not to band-to-band transitions, while $E_{g} \sim 11 \mathrm{eV}$ must be interpreted as the actual band-gap energy. ${ }^{27}$ Following this interpretation, absorption between $E_{0}$ and $E_{g}$ is mainly related to excitons and defect formation upon exposure to light in this spectral range is expected to be mainly driven by exciton dynamics. ${ }^{1-3,19,27,30,31}$

We report here experimental data evidencing the ability of VUV synchrotron radiation with photon energy above $E_{0}$ to generate luminescent $\mathrm{NBOHC}$ centers in extremely high concentrations near the surface of an ordinary silica sample. While these results bear some practical relevance related to the use of synchrotron as a photoexcitation source for silica, they also evidence previously unknown aspects of the generation processes of defects in silica by VUV radiation and of the excitation mechanisms of the $1.9 \mathrm{eV}$ luminescence characteristic of NBOHC defects. 


\section{EXPERIMENTAL SECTION}

We performed measurements on two "wet" synthetic commercial amorphous $\mathrm{SiO}_{2}$ samples (nickname: Suprasil311, Heraeus Quarzglas), manufactured by hydrolysis of $\mathrm{SiCl}_{4}$ in a $\mathrm{H}_{2} / \mathrm{O}_{2}$ flame, highly pure (concentration of metallic impurities below $1 \mathrm{ppm}(\mathrm{ppm})$ by weight) except for the presence of $\mathrm{Si}-\mathrm{OH}$ impurities in a concentration of $[\mathrm{Si}-\mathrm{OH}]=2.8 \times 10^{19} \mathrm{~cm}^{-3} .32 \quad$ Specimens were $5 \times 5 \times 0.5 \mathrm{~mm}^{3}$ sized, as obtained by cut of a single $50 \times 5 \times 0.5 \mathrm{~mm}^{3}$ slab. One of the two samples, hereafter referred to as $W_{0}$, was used as-received. This sample features no detectable luminescence or absorption signals before the experiment. The second sample, named $W_{\beta}$ and initially identical to $W_{0}$, was prepared for the experiment by irradiation with $\beta$ rays performed in a Van de Graaff accelerator (2.5 MeV electrons energy) with a $1.2 \cdot 10^{6} \mathrm{kGy}$ dose. Such a treatment induces in the sample the formation of several point defects, the main being the $E^{\prime}$ center and the $\mathrm{NBOHC}$, easily detectable by their characteristic 5.8 and $4.8 \mathrm{eV}$ absorption bands. Preliminary photoluminescence (PL) measurements on the $1.9 \mathrm{eV}$ emission band of the NBOHC excited at $4.8 \mathrm{eV}$ allowed to estimate its concentration $[\mathrm{NBOHC}]=1.2 \times 10^{18} \mathrm{~cm}^{-3}$ by comparison with a reference sample where the intensity of the $2.0 \mathrm{eV}$ absorption band of NBOHC could be measured. The absorption coefficient at $4.8 \mathrm{eV}$ was measured to be $8 \mathrm{~cm}^{-1}$. For comparison purposes, we performed measurements also on an as-grown "dry" synthetic $\mathrm{SiO}_{2}$ sample (commercial nickname: Suprasil F300, trademark of Heraeus Quartzglas), produced by reaction of $\mathrm{O}_{2}$ with $\mathrm{SiCl}_{4}$ in water-free plasma. This sample, hereafter referred to as $D_{0}$, features a very low concentration of $\mathrm{Si}-\mathrm{OH}$ impurities $\left([\mathrm{Si}-\mathrm{OH}]<8 \times 10^{16} \mathrm{~cm}^{-3}\right) .{ }^{32}$

We acquired PL spectra under excitation with synchrotron radiation at the SUPERLUMI experimental station in DESYHamburg (HASYLAB-Beamline I). In the experiments described in this paper, synchrotron radiation serves both as an irradiation source able to generate $\mathrm{NBOHC}$, and as a photoexcitation source of their $1.9 \mathrm{eV}$ emission band. The excitation wavelength was selected via a $2 \mathrm{~m}$ monochromator allowing an excitation bandwidth of $0.3 \mathrm{~nm}$. The cross section of the synchrotron beam was about $2.5 \mathrm{~mm}^{2}$. For the purposes of this experiment it is relevant to know the photon flux of the beamline at the sample position. From beamline's calibration data, ${ }^{33}$ its order of magnitude in our experimental conditions can be estimated to fall between $10^{13}-10^{14}$ photons $/\left(\mathrm{cm}^{2} \mathrm{~s}\right)$ at $E=11 \mathrm{eV}$. The emission spectrum was detected in front-face geometry with the sample in high vacuum $\left(10^{-9}-10^{-8}\right.$ mbar). The signal was dispersed by a 300 lines $/ \mathrm{mm}$ monochromator with $500 \mathrm{~nm}$ blaze, and detected by a charge coupled camera cooled by liquid $\mathrm{N}_{2}$. The emission bandwidth was $10 \mathrm{~nm}$. Each reported spectrum was corrected for the response and dispersion of the detecting system and scaled for the intensity of the synchrotron beam at the exciting photon energy. The exciting spectrum was estimated prior to the experiments by recording the excitation profile of a sodium salycilate reference sample. Temperature was controlled by a liquid He flow cryostat.

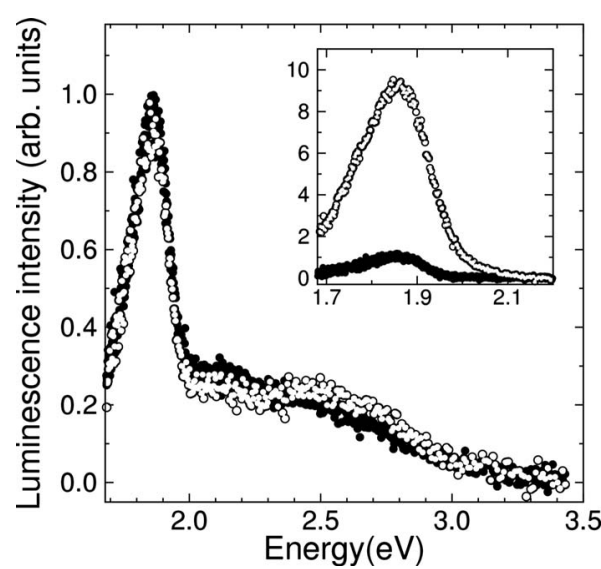

FIG. 1. Photoluminescence signal measured at $\mathrm{T}=10 \mathrm{~K}$ in asgrown (full symbols) $W_{0}$ and $\beta$ irradiated (empty symbols) $W_{\beta}$ silica samples under excitation by synchrotron light of $11.02 \mathrm{eV}$ photon energy. Inset: signal measured in the two samples under $4.8 \mathrm{eV}$ excitation.

\section{RESULTS}

The as-grown $W_{0}$ sample was cooled down to $\mathrm{T}=10 \mathrm{~K}$ and excited by synchrotron radiation with $11.02 \mathrm{eV}$ photon energy, falling above the absorption edge of amorphous silica. Under these conditions, the sample emits a composite luminescence signal, shown in Fig. 1, consisting in a well resolved peak at $1.86 \pm 0.02 \mathrm{eV}$ with $0.20 \pm 0.03$ full width at half maximum (FWHM) and a broad band centered at $2.5 \mathrm{eV}$. The spectral features of the $1.86 \mathrm{eV}$ peak are consistent with the characteristic emission signal of the NBOHC in silica ${ }^{7}$ hereafter this signal will be simply referred to as the $1.9 \mathrm{eV}$ band. Based on literature, the broad emission signal peaked at $2.5 \mathrm{eV}$ can be attributed to self-trapped-exciton (STE) luminescence. ${ }^{1,27}$ After this first measurement performed under above-edge excitation, the $W_{0}$ sample showed the $1.9 \mathrm{eV}$ signal also under $4.8 \mathrm{eV}$ excitation, as reported in the inset of the same figure. The intensity of the band was found to be roughly the same (1.0 arb. units peak value) under the two excitations, while the $2.5 \mathrm{eV}$ band was not detected under $4.8 \mathrm{eV}$ excitation. It is worth noting that before exposing the sample to above-edge light, the signal of the NBOHC excited at $4.8 \mathrm{eV}$ was absent in $W_{0}$, as usual in as-grown silica. In other words, the $1.9 \mathrm{eV}$ emission appeared in this sample only after the first exposure to aboveedge light.

Differently from the $W_{0}$ specimen, the irradiated $W_{\beta}$ sample already featured an intense $1.9 \mathrm{eV}$ luminescence band under $4.8 \mathrm{eV}$ excitation before any experiment performed with above-edge excitation. When $W_{\beta}$ was subjected to the same sequence of measurements performed on $W_{0}$, the following results were obtained: upon $11.02 \mathrm{eV}$ excitation, $W_{\beta}$ showed an emission spectrum with similar spectral features and almost identical intensity to that detected in the $W_{0}$ sample (Fig. 1). By contrast, when excited at $4.8 \mathrm{eV}$, the intensity of the $1.9 \mathrm{eV}$ band observed in $W_{\beta}$ was about nine times higher than that detected in $W_{0}$ (inset of Fig. 1).

We studied the excitation profile of these PL signals by recording the emission spectra in $W_{0}$ upon excitation at sev- 


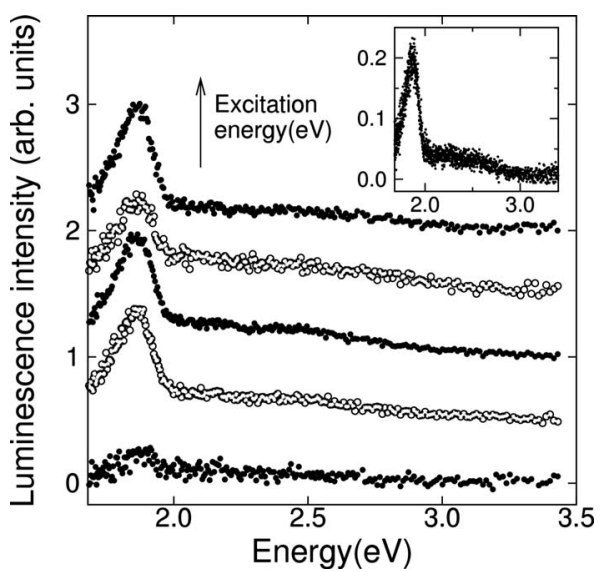

FIG. 2. Photoluminescence signals measured at $\mathrm{T}=10 \mathrm{~K}$ in $W_{0}$ sample under excitation by synchrotron light of $8.71,10.25,11.02$, 13.33, and $14.1 \mathrm{eV}$ photon energies. Spectra were vertically shifted for the sake of clarity. Inset: signal detected in $D_{0}$ sample by 10.25 $\mathrm{eV}$ excitation.

eral photon energies $E_{\text {exc }}$ (Fig. 2) from 8 to $14 \mathrm{eV}$. We observed that the excitation profile features a threshold energy of $\sim 8.7 \mathrm{eV}$, below which no emission is observed (except when exciting within the 4.8 or $6.4 \mathrm{eV}$ absorption bands of the defect). Above the threshold, both the $1.9 \mathrm{eV}$ and the 2.5 $\mathrm{eV}$ bands grow with $E_{\text {exc }}$, most of the growth occurring in the $8.7 \mathrm{eV}<E_{\text {exc }}<10 \mathrm{eV}$ interval. Only weak variations are then observed for $E_{\text {exc }}>10 \mathrm{eV}$. Also the ratio between the 1.9 and $2.5 \mathrm{eV}$ components weakly depends on $E_{\text {exc }}$. In the inset of Fig. 2 we report the luminescence spectrum measured at $\mathrm{T}=10 \mathrm{~K}$ on the $D_{0}$ sample upon $10.25 \mathrm{eV}$ excitation. The emission signal has a similar shape to that observed in the $W_{0}$ sample, with a $\sim 4$ times lower intensity.

In Fig. 3 we report the temperature dependence of the 1.9 and $2.5 \mathrm{eV}$ bands excited at $11.02 \mathrm{eV}$, as obtained by measuring the emission spectrum of the $W_{0}$ sample at several temperatures. The thermal quenching of both signals appears

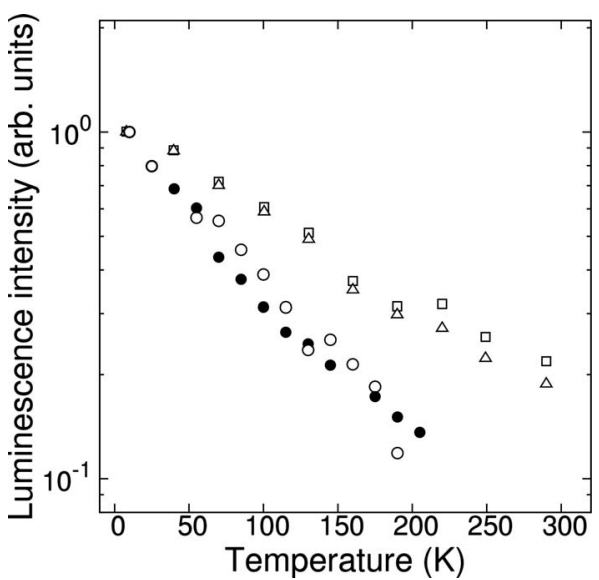

FIG. 3. Intensity of the $1.9 \mathrm{eV}$ (empty circles) and $2.5 \mathrm{eV}$ (full circles) luminescence signals detected in as-grown silica $W_{0}$ under excitation at $11.02 \mathrm{eV}$ as a function of temperature. Intensity of the $1.9 \mathrm{eV}$ signal as measured upon $4.8 \mathrm{eV}$ excitation (triangles) and $6.4 \mathrm{eV}$ excitation (squares). Data were normalized to the value measured at $\mathrm{T}=10 \mathrm{~K}$. to be active since the lowest measurement temperature. The $1.9 \mathrm{eV}$ signal decreases by a factor of $\sim 7$ when temperature grows from 10 to $200 \mathrm{~K}$. The temperature dependencies of the 1.9 and $2.5 \mathrm{eV}$ signals can be compared by normalizing both of them to the respective values detected at $\mathrm{T}=10 \mathrm{~K}$. Such a comparison evidences a close agreement between the two curves. Contrastingly, the latter are different from the ordinary temperature dependence of the NBOHC signal, defined by the measurement of the $1.9 \mathrm{eV}$ band under 4.8 or $6.4 \mathrm{eV}$ (or $2.0 \mathrm{eV}$, not reported) excitation. Indeed, by such a measurement we observe the $1.9 \mathrm{eV}$ signal decreasing only by a factor of $\sim 3$ in the same temperature interval, and by a factor of $\sim 5$ when going up to $300 \mathrm{~K}$. In regard to the STE emission at $2.5 \mathrm{eV}$, both its excitation threshold (Fig. 2) at about $8.7 \mathrm{eV}$, and the temperature dependence (Fig. 3) turn out to be consistent with literature data. ${ }^{27}$ The fact that the thermal quenching of STE emission begins from $10 \mathrm{~K}$ without featuring a lower threshold temperature, is typical of disordered materials such as amorphous $\mathrm{SiO}_{2}$, while it is not observed in crystalline $\mathrm{SiO}_{2} \cdot{ }^{27}$ This property suggests that a wide statistical distribution of activation energies controls the nonradiative decay process responsible for thermal quenching.

\section{DISCUSSION}

The appearance (Fig. 1) of the characteristic NBOHC luminescence signal at $1.9 \mathrm{eV}$ upon exposure to $11 \mathrm{eV}$ light, in an as-grown silica previously not showing any detectable luminescence activity, leads to two conclusions: (a) VUV light above the absorption edge triggers the generation of stable NBOHC defects in the silica matrix, as demonstrated by their subsequent observation upon $4.8 \mathrm{eV}$ excitation (inset of Fig. 1); (b) above-edge light is also able to excite the $1.9 \mathrm{eV}$ emission of NBOHC defects. The result (b) can be reasonably interpreted as an evidence of NBOHC being generated by above-edge light in their excited electronic state, which is a common situation in molecule photodissociation processes. $^{34,35}$ In this sense, the emission at $1.9 \mathrm{eV}$ upon 9-14 eV excitation (Fig. 2) can be regarded as a probe of ongoing generation of NBOHC defects. Given the order of magnitude of the absorption coefficient of silica at $11 \mathrm{eV}$, $\alpha_{0} \sim 10^{6} \mathrm{~cm}^{-1},^{29}$ the penetration depth can be roughly estimated as the length after which the incoming $11 \mathrm{eV}$ beam is 10-fold attenuated: $d_{0}=2.3 \alpha_{0}^{-1}=23 \mathrm{~nm}$. This estimate should be considered as very approximate, also because of the possible occurrence of effects unaccounted for, such as the finite thickness of the polished layer being comparable with $\alpha_{0}^{-1}$.

Strong evidences have been reported in literature suggesting that the band-to-band transition threshold of silica is located at $E_{g} \sim 11 \mathrm{eV}$, and that absorption at $E \leq E_{g}$ is basically excitonic, rather than being due to production of free charges across the gap. ${ }^{1,27}$ According to this interpretation, nonradiative decay of excitons must be assumed here as the fundamental mechanism leading to $\mathrm{NBOHC}$ generation upon illumination with $11 \mathrm{eV}$ light. Existing data indicate that only a minor portion of excitons undergoes self-trapping (as indicated by the $\sim 0.01$ quantum yield of STE luminescence in silica), so as to become immobile at low temperatures and 
eventually decay by emitting the observed $2.5 \mathrm{eV}$ band. ${ }^{1,27,30}$ Most of the generated excitations remain free excitons (FE) until their energy is lost nonradiatively. Our data thus confirm generation of point defects as one of the nonradiative decay mechanisms of FE, although other nonluminescent defects (possibly $E^{\prime}$ centers) may be formed along with the NBOHC centers. The hypothesis of a NBOHC generation mechanism mediated by excitons could justify the outcome evidenced by Fig. 2: the intensity of the 1.9 and $2.5 \mathrm{eV}$ signals depend on excitation energy in a similar way, both featuring the same threshold energy at $8.7 \mathrm{eV}$ and their ratio weakly depending on $E_{e x c}$. Moreover, as visible in Fig. 3, the temperature dependence of the $1.9 \mathrm{eV}$ band as measured upon $11 \mathrm{eV}$ excitation is quite different from that observed under ordinary 4.8 or $6 . \mathrm{eV}$ excitation. This indicates the involvement of temperature-dependent nonradiative processes playing a role before the excited NBOHC defect is ultimately generated. On the other side, the temperature dependence of the $1.9 \mathrm{eV}$ emission excited at $11 \mathrm{eV}$ is in close agreement with that of the STE signal at $2.5 \mathrm{eV}$. Also this finding qualitatively suggests a link between excitons and NBOHC generation, possibly the fact that STE luminescence and the generation of excited NBOHCs are both driven by FE. Nevertheless, a more detailed understanding of radiative and nonradiative channels determining the temperature dependency of the two emissions would be needed to verify the validity of this scheme. It has been argued that in amorphous $\mathrm{SiO}_{2}$ the mobility of FEs at low temperatures is much lower than in crystalline $\mathrm{SiO}_{2}$, and limited below the penetration depth of above-edge exciting light. ${ }^{27}$ Here, this implies that the generation of $\mathrm{NBOHC}$ by $11 \mathrm{eV}$ radiation takes place only in the thin region of depth $d_{0}$ below the surface accessible by the $11 \mathrm{eV}$ photons. Data in Fig. 2 demonstrate that the threshold for photogeneration of NBOHC is $\sim 9 \mathrm{eV}$. In the particular case in which $\mathrm{NBOHC}$ is induced in the sample by photons between 9 and $10 \mathrm{eV}$, the value of $d_{0}$ is expected to be higher than $23 \mathrm{~nm}$, as the absorption coefficient of silica grows from $\sim 10^{5}$ to $\sim 10^{6} \mathrm{~cm}^{-1}$ in this spectral region. ${ }^{29}$ With this in mind, we will use $d_{0}$ calculated at $11 \mathrm{eV}$ as a reference value in the rest of the discussion, which is devoted to discussing in further detail the generation mechanism of the defect.

Nonradiative decay of excitons has already been proposed in literature as a leading mechanism for defect generation, especially when excitons arise from two-photon absorption of laser light. ${ }^{16,17,19,21,36}$ The observation (Fig. 3) of thermal quenching of STE luminescence since the lowest measurement temperature indicates significant nonradiative decay of excitons already at $10 \mathrm{~K}$, this being consistent with the formation of permanent defects at this temperature. When the exposure to $11 \mathrm{eV}$ photons is carried out on the irradiated $W_{\beta}$ sample (Fig. 1), a remarkably similar signal is measured. However, when exciting at $4.8 \mathrm{eV}$, the signal in $W_{\beta}$ is about nine times higher than in $W_{0}$. This can be explained as follows: in the initially defect-free $W_{0}$ sample, $11 \mathrm{eV}$ photons create a distribution of defects concentrated in a $d_{0}$ deep volume, which are responsible of the signal measured upon $4.8 \mathrm{eV}$ excitation. In $W_{\beta}$, these thin depth defects are generated as well, with very similar concentration and spatial distribution. Nevertheless, such a distribution of defects is su- perimposed to the pre-existing bulk NBOHCs pre-induced in $W_{\beta}$ by $\beta$ irradiation, and uniformly distributed in the whole $d_{\beta}=0.5 \mathrm{~mm}$ thickness. As a consequence, in the $W_{\beta}$ sample the pre-existing bulk defects overwhelmingly contribute to the signal excited at $4.8 \mathrm{eV}$, while the similarity between the signals excited at $11 \mathrm{eV}$ in the two samples indicates that the generation process of $\mathrm{NBOHC}$ in the $d_{0}$ deep volume occurs with the same efficiency in the two samples independently of the pre-existing defects in $W_{\beta}$.

To make this argument quantitative, we note that the emission signal measured upon excitation at photon energy $E$ with intensity $I(E)$ is given by:

$$
S(E)=y I(E)\left(1-e^{-\alpha(E) d}\right) \eta(E),
$$

where $\alpha(E)$ is the absorption coefficient, $\eta(E)$ the PL quantum yield, $y$ is a geometrical factor expressing the collecting efficiency of emitted photons, while $I(E)$ will be omitted hereafter since all emission spectra were scaled for excitation intensity. When exciting at $4.8 \mathrm{eV}$, for sake of simplicity Eq. (1) can be approximated by $S(4.8 \mathrm{eV}) \cong y \alpha(4.8 \mathrm{eV}) d \eta(4.8 \mathrm{eV}) \quad$ within a $21 \%$ accuracy. ${ }^{41}$ The absorption coefficient is related to defect concentration by: $\quad \alpha(4.8 \mathrm{eV})=\sigma(4.8 \mathrm{eV}) \times[\mathrm{NBOHC}]$, where the absorption cross section can be calculated from the oscillator strength $f=0.04$ to be $\sigma(4.8 \mathrm{eV})$ $=5.5 \times 10^{-18} \mathrm{~cm}^{-2} .{ }^{42}$ We can estimate the order of magnitude of the local concentration of NBOHC induced by synchrotron radiation in the $d_{0}$ deep volume, by comparing the detected intensity $S(4.8 \mathrm{eV})$ in the two samples. This quantity can be expressed by Eq. (1), setting $d$ for each sample to be the depth in which defects probed by $4.8 \mathrm{eV}$ excitation are distributed: $d=d_{0}=23 \mathrm{~nm}$ for $W_{0}$ and $d=d_{\beta}=0.5 \mathrm{~mm}$ for $W_{\beta}$. In this way we obtain:

$$
\begin{aligned}
\frac{S\left(4.8 \mathrm{eV}, W_{0}\right)}{S\left(4.8 \mathrm{eV}, W_{\beta}\right)} & \cong \frac{y \alpha\left(4.8 \mathrm{eV}, W_{0}\right) d_{0} \eta(4.8 \mathrm{eV})}{y \alpha\left(4.8 \mathrm{eV}, W_{\beta}\right) d_{\beta} \eta(4.8 \mathrm{eV})} \\
& =\frac{d_{0}[\mathrm{NBOHC}]_{W_{0}}}{d_{\beta}[\mathrm{NBOHC}]_{W_{\beta}}}
\end{aligned}
$$

The leftmost side of Eq. (2) equals $1 / 9$ from data in the inset of Fig. 1. Substituting also $[\mathrm{NBOHC}]_{W_{\beta}}=1.2 \times 10^{18} \mathrm{~cm}^{-3}$, we get $[\mathrm{NBOHC}]_{W_{0}} \sim 3 \times 10^{21} \mathrm{~cm}^{-3}$. This is a very high value corresponding to about $1 / 10$ of the concentration of tetrahedra in the silica matrix and to a mean NBOHC$\mathrm{NBOHC}$ distance as low as $0.7 \mathrm{~nm}$. This very approximate estimate has to be taken as an upper limit for NBOHC concentration. However, it clearly points to a picture in which the thin $\mathrm{SiO}_{2}$ layer is so highly damaged by synchrotron radiation that the local concentration of induced defects is orders of magnitude higher than that induced by high doses $\left(10^{6} \mathrm{kGy}\right)$ of $\beta$ radiation. It is worth noting that the local defect density $\left(\sim 10^{21} \mathrm{~cm}^{-3}\right)$ found in the subsurface layer is orders of magnitude higher than ever reported for silica irradiation experiments and may play an important role in a variety of processes such as synchrotron-induced surface 
evaporation and light-stimulated desorption. ${ }^{37} \mathrm{We}$ also note that the concentration of $\mathrm{NBOHC}$ formed in $W_{0}$ per unit surface can be calculated as $d_{0}[\mathrm{NBOHC}]_{W_{0}} \sim 7 \times 10^{15} \mathrm{~cm}^{-2}$, comparable with the total number of photons received per unit surface by the sample during a typical exposure time $\left(\sim 10^{2} \mathrm{~s}\right)$ in a measurement upon above-gap light. This suggests defect formation upon synchrotron irradiation to be very efficient (virtually approaching one defect per absorbed photon).

We finally discuss the precursor of NBOHC defects. Two generation processes are widely accepted in literature: (a) breaking of the oxygen-hydrogen bond on $\mathrm{Si}-\mathrm{OH}$ precursor sites and (b) rupture of $\mathrm{Si}-\mathrm{O}-\mathrm{Si}$ bonds leading to the correlated formation of $\mathrm{NBOHCs}$ and $E^{\prime}$ centers. ${ }^{18,38,39}$ Given the very high estimated local concentration of formed defects (compare with $[\mathrm{Si}-\mathrm{OH}]=2.8 \times 10^{19} \mathrm{~cm}^{-3}$ in $W_{0}$ prior to the experiment), and since most of the incoming $11 \mathrm{eV}$ photons are absorbed on ordinary matrix sites giving rise to excitonic transitions, one could simply conclude that $\mathrm{NBOHCs}$ are not generated in the thin layer by transformation of pre-existing $\mathrm{Si}-\mathrm{OH}$ precursors, but they are rather formed out from initially undefected matrix sites. This model is consistent with data obtained (inset of Fig. 2) on the dry $\mathrm{SiO}_{2}$ sample $D_{0}$. Since the $\mathrm{Si}-\mathrm{OH}$ concentration in $D_{0}$ is $\sim 350$ times lower than in $W_{0}$, the detection in $D_{0}$ of a signal $\sim 4$ times smaller than in $W_{0}$ appears to confirm that the main contribution to induced $\mathrm{NBOHC}$ defects arises from breakage of $\mathrm{Si}-\mathrm{O}-\mathrm{Si}$ bonds. ${ }^{43}$ So high a concentration of formed NBOHCs also suggests that in the subsurface layer most of the $\mathrm{Si}-\mathrm{O}-\mathrm{Si}$ sites are potential precursors for NBOHC generation. This is different from what is known for bulk silica, where it is generally assumed that only strained $\mathrm{Si}-\mathrm{O}-\mathrm{Si}$ bonds are efficient precursors for defect formation. ${ }^{1}$

It is worth noting, however, that the local concentration of $\mathrm{Si}-\mathrm{OH}$ impurities near the surface of the samples is not expected to be necessarily the same as in the bulk material. In particular, the $\mathrm{Si}-\mathrm{OH}$ concentration on the surface, or in a thin layer below the surface, could be higher than in the bulk, and even comparable for dry and wet materials. For instance, studies on finely divided and/or porous silica systems have pointed out that the surface concentration of $\mathrm{OH}$ groups can be equal to that of silicon atoms. ${ }^{40}$ So high a concentration is never found in the bulk of any $\mathrm{SiO}_{2}$ material. If this occurs, our results would still be consistent with generation of NBOHC occurring by breaking of $\mathrm{Si}-\mathrm{OH}$ precursors.

While present data do not allow to discern conclusively between the two possibilities, some indications can be drawn by optical absorption, laser-excited photoluminescence and electron spin resonance (ESR) measurements performed ex situ, i.e., after conclusion of the experimental session with synchrotron radiation, and removing the sample from the high vacuum sample chamber. We were not able to detect any appreciable variation within $10^{-2}$ optical densities of the absorption spectrum of $W_{0}$ and $W_{\beta}$ samples after exposure to synchrotron radiation. In contrast, $\mathrm{NBOHCs}$ were detected in the $W_{0}$ sample by PL excited by $4.8 \mathrm{eV}$ laser at room temperature, in a concentration $[\mathrm{NBOHC}]_{\text {ex situ }}=4 \times 10^{20} \mathrm{~cm}^{-3}$, about 7.5 times lower than estimated above during synchro- tron measurements. We believe the reduction to be due to passivation of near-surface defects by gaseous species, either present in the specimen and starting to diffuse when the temperature is raised (e.g., hydrogen produced together with $\mathrm{NBOHC}$ by $\mathrm{OH}$ breaking), or coming from ambient air when the sample is removed from vacuum. We remark that the value $[\mathrm{NBOHC}]_{e x \text { situ }}$ corresponds to an optical attenuation of $\alpha * d_{0}=5 \times 10^{-3}$ on the peak of the $4.8 \mathrm{eV}$ band that is smaller than our detection limit in absorption measurement. In regard to ESR measurements, we did not detect the signal of $E^{\prime}$ centers within our detection limit of roughly $10^{12}$ spins. This provides a clue to understand the generation mechanism of $\mathrm{NBOHC}$. In fact, rupture of $\mathrm{Si}-\mathrm{O}-\mathrm{Si}$ bonds would be expected to generate $\mathrm{NBOHC}$ and $E^{\prime}$ centers in the same concentration, inconsistent with the ESR results, since a concentration of $[\mathrm{NBOHC}]_{\text {ex situ }}=4 \times 10^{20} \mathrm{~cm}^{-3}$ corresponds to an absolute number $2 \times 10^{13}$ of defects, based on the width $d_{0}$ of the damaged layer and on the area of the irradiated spot. This result suggests generation from near-surface $\mathrm{Si}-\mathrm{OH}$ to be the prevalent formation mechanism of $\mathrm{NBOHC}$ by above-gap light, as opposed to generation from $\mathrm{Si}-\mathrm{O}-\mathrm{Si}$ bonds. While $\mathrm{Si}-\mathrm{O}-\mathrm{Si}$ breaking is generally regarded in literature as a natural outcome of nonradiative exciton decay, it is not obvious the mechanism by which $\mathrm{Si}-\mathrm{OH}$ can be broken by nonradiative decay of excitons formed by absorption of above-gap light. The exciton needs to be trapped on a $\mathrm{Si}-\mathrm{OH}$ impurity and decay by spending its energy in the breakage of the $\mathrm{O}-\mathrm{H}$ bond. The details of this process remain open at the moment.

\section{CONCLUSIONS}

We demonstrated that the exposure of amorphous $\mathrm{SiO}_{2}$ to above-edge synchrotron light at low temperature causes the formation of a remarkably high concentration of $\mathrm{NBOHC}$ defects in a thin layer extended for few $10^{-8} \mathrm{~m}$ below the surface. At the same time, we provide direct evidence that the $1.9 \mathrm{eV}$ emission of NBOHC is triggered by above-edge illumination. We interpret both findings by proposing that nonradiative decay of excitons induced by absorption of VUV light leads to the generation of $\mathrm{NBOHC}$ in the emitting excited electronic state. After generation, NBOHCs can be detected by observing their characteristic $1.9 \mathrm{eV}$ emission upon $4.8 \mathrm{eV}$ excitation. The combined result of ESR, synchrotron, and laser-excited PL measurements suggests exciton-driven breakage of near-surface $\mathrm{Si}-\mathrm{OH}$ groups to be the leading generation mechanism of NBOHCs.

\section{ACKNOWLEDGMENTS}

We acknowledge financial support received by DESY (Project No. II-20052090EC). We thank B. Boizot for taking care of $\beta$ irradiation at Ecole Polytechnique-Palaiseau (France). We are grateful to the LAMP research group (http://www.fisica.unipa.it/amorphous/) for support and enlightening discussions and to G. Stryganyuk for assistance in experimental sessions at HASYLAB. 
*fmessina@fisica.unipa.it

${ }^{1}$ Defects in $\mathrm{SiO}_{2}$ and Related Dielectrics: Science and Technology, edited by G. Pacchioni, L. Skuja, and D. L. Griscom (Kluwer Academic Publishers, USA, 2000).

${ }^{2}$ Structure and Imperfections in Amorphous and Crystalline Silicon Dioxide, edited by R. A. B. Devine, J.-P. Duraud, and E. Dooryhe (Wiley, UK, 2000).

${ }^{3}$ Silicon-based Materials and Devices, edited by H. S. Nalwa (Academic Press, USA, 2001).

${ }^{4}$ L. Skuja, H. Hosono, and M. Hirano, Proc. SPIE 4347, 155 (2001).

${ }^{5}$ D. L. Griscom, E. J. Friebele, and G. H. Siegel, Solid State Commun. 15, 479 (1974).

${ }^{6}$ M. Stapelbroek, D. L. Griscom, E. J. Friebele, and G. H. Sigel, Jr., J. Non-Cryst. Solids 32, 313 (1979).

${ }^{7}$ L. Skuja, J. Non-Cryst. Solids 179, 51 (1994).

${ }^{8}$ L. Skuja and K. Tanimura, J. Appl. Phys. 80, 3518 (1996).

${ }^{9}$ H. Hosono, K. Kajihara, T. Suzuki, Y. Ikuta, L. Skuja, and M. Hirano, Solid State Commun. 122, 117 (2002).

${ }^{10}$ M. Cannas and F. M. Gelardi, Phys. Rev. B 69, 153201 (2004).

${ }^{11}$ M. Cannas, L. Vaccaro, and B. Boizot, J. Non-Cryst. Solids 352, 203 (2006).

${ }^{12}$ L. Skuja, T. Suzuki, and K. Tanimura, Phys. Rev. B 52, 15208 (1995).

${ }^{13}$ T. Suzuki, L. Skuja, K. Kajihara, M. Hirano, T. Kamiya, and H. Hosono, Phys. Rev. Lett. 90, 186404 (2003).

${ }^{14}$ L. Vaccaro, M. Cannas, and R. Boscaino, Solid State Commun. 146, 148 (2008).

${ }^{15}$ L. Vaccaro, M. Cannas, and V. Radzig, Phys. Rev. B 78, 233408 (2008).

${ }^{16}$ T. E. Tsai and D. L. Griscom, Phys. Rev. Lett. 67, 2517 (1991).

${ }^{17}$ H. Nishikawa, R. Nakamura, Y. Ohki, and Y. Hama, Phys. Rev. B 48, 15584 (1993).

${ }^{18}$ H. Imai, K. Arai, J. Isoya, H. Hosono, Y. Abe, and H. Imagawa, Phys. Rev. B 48, 3116 (1993).

${ }^{19}$ R. A. B. Devine, Nucl. Instrum. Methods Phys. Res. B 91, 378 (1994).

${ }^{20}$ K. Kajihara, L. Skuja, M. Hirano, and H. Hosono, Phys. Rev. Lett. 89, 135507 (2002).

${ }^{21}$ N. Fukata, Y. Yamamoto, K. Murakami, M. Hase, and M. Kitajima, Appl. Phys. Lett. 83, 3495 (2003).

${ }^{22}$ S. O. Kucheyev and S. G. Demos, Appl. Phys. Lett. 82, 3230 (2003).

${ }^{23}$ A. Zoubir, C. Rivero, R. Grodsky, K. Richardson, M. Richardson, T. Cardinal, and M. Couzi, Phys. Rev. B 73, 224117 (2006).
${ }^{24}$ F. Messina and M. Cannas, J. Phys.: Condens. Matter 18, 9967 (2006).

${ }^{25}$ F. Messina and M. Cannas, J. Phys.: Condens. Matter 20, 275210 (2008).

${ }^{26}$ K. Kajihara, M. Hirano, L. Skuja, and H. Hosono, Phys. Rev. B 78, 094201 (2008).

${ }^{27}$ A. N. Trukhin, J. Non-Cryst. Solids 149, 32 (1992).

${ }^{28}$ C. Bosio and W. Czaja, Europhys. Lett. 24, 197 (1993).

${ }^{29}$ G. L. Tan, M. F. Lemon, D. J. Jones, and R. H. French, Phys. Rev. B 72, 205117 (2005).

${ }^{30}$ P. N. Saeta and B. I. Greene, Phys. Rev. Lett. 70, 3588 (1993).

${ }^{31}$ S. Ismail-Beigi and S. G. Louie, Phys. Rev. Lett. 95, 156401 (2005).

${ }^{32}$ Heraeus Quartzglas, Hanau, Germany, catalog POL-0/102/E (2001).

${ }^{33}$ G. Zimmerer, Radiat. Meas. 42, 859 (2007).

${ }^{34}$ W. A. Jackson and R. J. Cody, J. Chem. Phys. 61, 4183 (1974).

${ }^{35}$ H. Xu, Y. Guo, S. Liu, X. Ma, D. Dai, and G. Sha, J. Chem. Phys. 117, 5722 (2002).

${ }^{36}$ H. Imai and H. Hirashima, J. Non-Cryst. Solids 179, 202 (1994).

${ }^{37}$ H. Akazawa, Phys. Rev. B 52, 12386 (1995).

${ }^{38}$ R. A. B. Devine and J. Arndt, Phys. Rev. B 39, 5132 (1989).

${ }^{39}$ L. Vaccaro, M. Cannas, B. Boizot, and A. Parlato, J. Non-Cryst. Solids 353, 586 (2007).

${ }^{40}$ L. T. Zhuravlev, Langmuir 3, 316 (1987).

${ }^{41}$ The absorption coefficient at $4.8 \mathrm{eV}$ in $W_{\beta}$ was measured to be $8 \mathrm{~cm}^{-1}$, slightly higher than expected $\left(6.6 \mathrm{~cm}^{-1}\right)$ from the known cross section of $\mathrm{NBOHC}$, due to minor contributions associated to other defects. This value of $\alpha$ yields an attenuation of $\alpha(4.8 \mathrm{eV}) d_{\beta} \sim 0.4$. With this value, one gets $[1-\exp (-\alpha d)]$ $\sim 0.33$. Since $0.4 / 0.33=1.21$, one can estimate the approximation of low absorption for $W_{\beta}$ to be correct within $21 \%$, acceptable for the present purposes. All the more so, this is true for $W_{0}$, since data in the inset of Fig. 1 imply the attenuation of $W_{0}$ at $4.8 \mathrm{eV}$ to be 9 times smaller.

${ }^{42}$ The $f$ value of the $4.8 \mathrm{eV}$ absorption band is proportional to that $\left(1.9 \times 10^{-4}\right)$ of the $2.0 \mathrm{eV}$ band, ${ }^{14}$ experimentally derived from the radiative decay constant of the $1.9 \mathrm{eV}$ luminescence, the proportionality coefficient being the ratio between the absorption band integrals $(\sim 200) .{ }^{11}$

${ }^{43}$ The small reduction in intensity from $W_{0}$ to $D_{0}$ may be due to some factor unaccounted for in data analysis. In particular, we expect the unchecked status of the surface to be critical in determining the intensity of this PL signal arising from such a thin layer. Two materials manufactured by different techniques are not expected to be identical as concerns surface roughness, this potentially leading to fluctuations in signal intensity. 